\title{
Spin-Orbital Physics in Transition Metal Oxides
}

\author{
AndrZEJ M. Oleś \\ Marian Smoluchowski Institute of Physics, Jagellonian University \\ Reymonta 4, PL-30-059 Kraków, Poland \\ and Max-Planck-Institut für Festkörperforschung \\ Heisenbergstr. 1, D-70569 Stuttgart, Germany
}

\begin{abstract}
We present the main features of the spin-orbital superexchange which describes the magnetic and optical properties of the Mott insulators with orbital degrees of freedom. In contrast to the SU(2) symmetry of spin superexchange, the orbital part of the superexchange obeys the lower cubic symmetry of the lattice and is intrinsically frustrated. This intrinsic frustration and spin-orbital entanglement induce enhanced quantum fluctuations, and we point out a few situations where this leads to disordered states. Strong coupling between the spin and orbital degrees of freedom is discussed on the example of the $\mathrm{RVO}_{3}$ perovskites, with $\mathrm{R}$ standing for rare-earth ion, $\mathrm{La}, \ldots, \mathrm{Lu}$. We explain the observed evolution of the orbital $T_{\mathrm{OO}}$ and Néel $T_{\mathrm{N} 1}$ transition temperature in the $\mathrm{RVO}_{3}$ series with decreasing ionic radius $r_{\mathrm{R}}$. A few open problems and the current directions of research in the field of spin-orbital physics are pointed out.
\end{abstract}

PACS numbers: 75.10.Jm, 75.30.Et, 03.67.Mn, 61.50.Ks

\section{Orbital versus spin superexchange}

In recent years the physical properties of Mott (or charge transfer) insulators are in the focus of interest in the condensed matter theory [1]. In order to develop theoretical understanding of complex phenomena in doped correlated insulators, including high temperature superconductivity in the cuprates and the colossal magnetoresistance (CMR) in the manganites, it is necessary to describe first the undoped materials, such as $\mathrm{La}_{2} \mathrm{CuO}_{4}$ and $\mathrm{LaMnO}_{3}$. In both cases the local Coulomb interactions (Hubbard $U$ ) is large and suppresses charge fluctuations, leading to low-energy effective Hamiltonians with superexchange interactions which stabilize antiferromagnetic $(\mathrm{AF})$ spin order at low temperature $[2,3]$. However, these two compounds are qualitatively quite different. On the one hand, the degeneracy of partly filled $e_{\mathrm{g}}$ orbitals is lifted in $\mathrm{La}_{2} \mathrm{CuO}_{4}$ by the tetragonal distortions of $\mathrm{CuO}_{6}$ octahedra, resulting in two-dimensional (2D) AF superexchange of $S=1 / 2$ holes in $x^{2}-y^{2}$ orbitals of $\mathrm{Cu}^{2+}$ ions. On the other hand, in $\mathrm{LaMnO}_{3} e_{\mathrm{g}}$ orbital degeneracy plays a fundamental role and, together with the Jahn-Teller (JT) distortions, is required to understand the origin of the anisotropic $A$-type $\mathrm{AF}(A-\mathrm{AF})$ order [4]. As realized already three decades ago [2], in this latter case the orbital degrees of freedom, which are described by the components

$$
|x\rangle \equiv \frac{1}{\sqrt{2}}\left(x^{2}-y^{2}\right), \quad|z\rangle \equiv \frac{1}{\sqrt{2}}\left(3 z^{2}-r^{2}\right)
$$

of pseudospin $\tau=1 / 2$ and contribute explicitly to the structure of the superexchange. Thus they have to be included on equal footing with ionic spins in a spin- orbital superexchange model. In the last two decades several new concepts were developed, such as enhanced quantum fluctuations due to orbital degrees of freedom which participate in joint spin-orbital excitations [5], and spin-orbital entanglement which occurs in cases when spin and orbital operators cannot be decoupled from each other [6]. The actual physical problems in this emerging and rapidly developing field were reviewed in the Focus Issue Orbital Physics in New Journal of Physics [7] a few years ago. Here we want to focus on a few representative recent developments in the field of spin-orbital physics.

Before we will discuss a few consequences of realistic spin-orbital models, let us consider first purely orbital superexchange interactions, as realized in ferromagnetic (FM) 2D systems, such as $\mathrm{K}_{2} \mathrm{CuF}_{4}$, or in $\mathrm{FM} a b$ planes of three-dimensional (3D) perovskites, either in $\mathrm{KCuF}_{3}$ or in $\mathrm{LaMnO}_{3}$. The superexchange originates from charge $d_{i}^{9} d_{j}^{9} \rightleftharpoons d_{i}^{8} d_{j}^{10}$ excitations (with $d_{i}^{9} \equiv t_{2 \mathrm{~g}}^{6} e_{\mathrm{g}}^{3}, d_{i}^{8} \equiv t_{2 \mathrm{~g}}^{6} e_{\mathrm{g}}^{2}$ ) in the first case (for $\mathrm{Cu}^{2+}$ ions), and from $d_{i}^{4} d_{j}^{4} \rightleftharpoons d_{i}^{3} d_{j}^{5}$ (with $d_{i}^{4} \equiv t_{2 \mathrm{~g}}^{3} e_{\mathrm{g}}, d_{i}^{3} \equiv t_{2 \mathrm{~g}}^{3}$, and $d_{i}^{5} \equiv t_{2 \mathrm{~g}}^{3} e_{\mathrm{g}}^{2}$ ) excitations in the second one (for $\mathrm{Mn}^{3+}$ ions) [8]. These charge excitations can be transformed away by applying a similar canonical transformation to the one leading to the $t-J$ model [9], commonly used to describe the superconducting cuprates. For the FM compound only high-spin excited states contribute, and in the strong-coupling regime $(t \ll U)$ one obtains the effective low-energy Hamiltonian describing orbital superexchange between pseudospins $\tau=1 / 2$ due to $e_{\mathrm{g}}$ electrons (holes in $\mathrm{KCuF}_{3}$ ) in a cubic crystal $[5,10]$ : 


$$
\mathcal{H}_{0}=H_{J}+H_{z},
$$

the first term $H_{J}$ in Eq. (2) describes superexchange interaction which depends on the superexchange constant

$$
J=\frac{4 t^{2}}{U}
$$

[here $t$ is the $(d d \sigma)$ hopping element between two $|z\rangle$ orbitals along the $c$ axis, and $U$ is the intraorbital Coulomb interaction], and on Hund's exchange element

$$
\eta=\frac{J_{\mathrm{H}}}{U}
$$

This interaction parameterizes the multiplet structure of transition metal ions when anisotropy of the Coulomb and exchange elements is neglected [8]. In the simplest case of $d^{9}$ system, the singlet-triplet splitting between the two lowest $d^{8}$ excited states is $2 \eta[11]$.

Following Ref. [10], we will consider below a 3D (perovskite) system with FM spin order and orbital interactions described by the Hamiltonian (2). The origin of intrinsic frustration in the orbital superexchange is best realized by considering its form

$$
H_{J}=\frac{1}{2} J r_{1} \sum_{\langle i j\rangle}\left(\tau_{i}^{(\gamma)} \tau_{j}^{(\gamma)}-\frac{1}{4}\right),
$$

where $\gamma=a, b, c$ refers to the cubic axes, and $r_{1}=$ $1 /(1-3 \eta)$ follows from the energy of the high-spin charge excitation [8]. The pseudospin operators are defined as follows:

$$
\tau_{i}^{(a, b)}=\frac{1}{4}\left(-\sigma_{i}^{z} \pm \sqrt{3} \sigma_{i}^{x}\right), \quad \tau_{i}^{(c)}=\frac{1}{2} \sigma_{i}^{z},
$$

where $\sigma_{i}^{x(z)}$ are the Pauli matrices and sign $+(-)$ is selected for a bond $\langle i j\rangle$ along $a(b)$ axis. The pseudospin interactions $\propto \tau_{i}^{(\alpha)} \tau_{j}^{(\alpha)}$ favor alternating orbitals (AOs) on each bond. They are fundamentally different from the $\mathrm{SU}(2)$-symmetric spin interactions, as they: (i) obey only lower cubic symmetry, (ii) are Ising-like, having only one component of the pseudospin interaction which favors pairs of orbitals oriented along the bond (z-like) and in the plane perpendicular to the bond ( $x$-like), and (iii) change their form when the cubic direction is changed. The interactions (5) look to be classical, but in fact due to the form of the pseudospin $e_{\mathrm{g}}$ operators (6) they are not. However, the quantum corrections generated by them are rather small [10].

The second term $H_{z}$ in Eq. (2) stands for the orbital splitting (by $J \varepsilon_{z}$ ), and follows from finite crystal field at either $\mathrm{Cu}^{2+}$ or $\mathrm{Mn}^{3+}$ ions:

$$
H_{z}=-J \varepsilon_{z} \sum_{i} \tau_{i}^{(c)} \text {. }
$$

The crystal-field splitting favors electron (hole) occupancy in $x(z)$ orbital for $\varepsilon_{z}>0$ or $\left(\varepsilon_{z}<0\right)$. This term is induced by static distortions in the tetragonal field and is of particular importance in 2D systems, e.g. in the high- $T_{\mathrm{c}}$ cuprates.

The directional nature of pseudospin orbital interactions is responsible for their intrinsic frustration $[5,12]$. In fact, the pair of orbitals which would minimize the energy for a bond $\langle i j\rangle$ is different for each cubic axis.
Therefore, while for a single bond the minimal energy of $-\frac{1}{4} J$ can easily be obtained by selecting a pair of orthogonal orbitals on both sites, such as $|z\rangle$ and $|x\rangle$ orbital for a bond along the $c$ axis (let us note that this corresponds to the Ising superexchange interaction as only one orbital is active in intersite charge excitations), this is no longer possible for a 2D (or 3D) system. Thus, unlike in spin systems, the tendency towards disorder (quantum orbital liquid) is enhanced with increasing system dimension [13].

The essence of orbital frustration which characterizes the $e_{\mathrm{g}}$ orbital superexchange (5) is captured by the 2D compass model, originally developed as a model for the Mott insulators [2]. Intersite interactions in the compass model are described by products $\tau_{i}^{\alpha} \tau_{j}^{\alpha}$ of pseudospin components, where $\alpha=x, y, z$, rather than by a scalar product $\boldsymbol{\tau}_{i} \cdot \boldsymbol{\tau}_{j}$ :

$$
\tau_{i}^{x}=\frac{1}{2} \sigma_{i}^{x}, \quad \tau_{i}^{y}=\frac{1}{2} \sigma_{i}^{y}, \quad \tau_{i}^{z}=\frac{1}{2} \sigma_{i}^{z},
$$

where $\alpha=x, y, z$, rather than by a scalar product $\boldsymbol{\tau}_{i} \cdot \boldsymbol{\tau}_{j}$. In the $2 \mathrm{D}$ case the $\tau_{i}^{x} \tau_{j}^{x}$ interactions for bonds $\langle i j\rangle$ along the $a$ axis compete with the $\tau_{i}^{z} \tau_{j}^{z}$ ones along the $b$ axis [14]. Recently certain aspects of this model were investigated by analytic [14] and numerical $[15,16]$ methods. Despite its closeness to ordinary models used in quantum magnetism, there is no ordered phase with finite magnetization. Thus, this case is qualitatively different from the frustrated Ising interactions on the 2D square lattice, where it was proven rigorously that magnetic order develops below certain transition temperature which depends on the ratio of frustrated to nonfrustrated plaquettes [17]. A competition of pseudospin interactions along different directions results instead in intersite correlations similar to the anisotropic $X Y$ model, and competition between two types of Ising-like order generates a quantum phase transition with high degeneracy of the ground state when all interactions have the same strength [15].

It is interesting to note that a similar quantum phase transition exists also in the one-dimensional (1D) compass model [18], when both above interactions alternate along the chain $\left(N^{\prime}=N / 2\right.$ is the number of unit cells):

$$
\mathcal{H}_{1 \mathrm{D}}=\sum_{i=1}^{N^{\prime}}\left\{J_{x} \tau_{2 i-1}^{x} \tau_{2 i}^{x}+J_{z} \tau_{2 i}^{z} \tau_{2 i+1}^{z}\right\} .
$$

This model was solved exactly in the whole range of $\left\{J_{x}, J_{z}\right\}$ parameters [18] by mapping to the exactly solvable quantum Ising model [19] in different subspaces (see also this volume [20]). Equal coupling constants $J_{x}=J_{z}=J$ correspond to the quantum critical point, where the orbital liquid emerges from two different disordered phases, with hidden order of pairs of pseudospins on every second bond. Presented here 1D model (9) provides thus a beautiful example of a first order quantum phase transition.

\section{Spin-orbital models and exotic liquid states}

The structure of spin-orbital superexchange involves interactions between $\mathrm{SU}(2)$-symmetric spin scalar products $\boldsymbol{S}_{i} \cdot \boldsymbol{S}_{j}$ on each bond $\langle i j\rangle$ connecting two nearest- 
-neighbor transition metal ions, each one coupled to orbital operators $\left\{\boldsymbol{\tau}_{i}, \boldsymbol{\tau}_{j}\right\}$ which obey only much lower symmetry (at most cubic for a cubic lattice) and its general form is [8]:

$$
\mathcal{H}_{J}=J \sum_{\langle i j\rangle}\left\{\hat{\mathcal{J}}_{i j}^{(\gamma)}\left(\boldsymbol{S}_{i} \cdot \boldsymbol{S}_{j}+S^{2}\right)+\hat{\mathcal{K}}_{i j}^{(\gamma)}\right\} .
$$

The orbital operators $\hat{\mathcal{J}}_{i j}^{(\gamma)}$ and $\hat{\mathcal{K}}_{i j}^{(\gamma)}$ involve the active orbitals on each bond $\langle i j\rangle$ along direction $\gamma$, either $e_{\mathrm{g}}$ or $t_{2 \mathrm{~g}}$, which participate in $d_{i}^{n} d_{j}^{n} \rightleftharpoons d_{i}^{n+1} d_{j}^{n-1}$ virtual excitations, and thus have the symmetry of the lattice (e.g. cubic in the perovskites). The superexchange model (10) consists of several terms which originate from different charge excitations. This feature made it possible to relate the averages of these different excitations to the spectral weights in the optical spectroscopy [21], and this serves now as a theoretical tool to explain the observed anisotropy and temperature dependence of the spectral weights in the optical spectra [8].

The best known spin-orbital model is the KugelKhomskii model for $d^{9} \mathrm{Cu}^{2+}$ ions which involves the $e_{\mathrm{g}}$ orbital operators (6) and includes both FM and AF spin interactions, depending on whether the above charge excitation (for $n=9$ ) involves the high-spin (triplet) or one of the low-spin (singlet) $d^{8}$ states. These excitations are parameterized by the coefficients $r_{1}=1 /(1-3 \eta)$, $r_{2}=1 /(1-\eta)$ and $r_{4}=1 /(1+\eta)$. Using compact notation of Ref. [11] it can be written as follows:

$$
\begin{aligned}
& \mathcal{H}_{J}\left(d^{9}\right)=\frac{1}{2} J \sum_{\langle i j\rangle}\left\{\left[-r_{1}\left(\boldsymbol{S}_{i} \cdot \boldsymbol{S}_{j}+\frac{3}{4}\right)\right.\right. \\
& \left.+r_{2}\left(\boldsymbol{S}_{i} \cdot \boldsymbol{S}_{j}-\frac{1}{4}\right)\right]\left(\frac{1}{4}-\tau_{i}^{(\gamma)} \tau_{j}^{(\gamma)}\right) \\
& \quad+\left(r_{2}+r_{4}\right)\left(\boldsymbol{S}_{i} \cdot \boldsymbol{S}_{j}-\frac{1}{4}\right)\left(\frac{1}{2}-\tau_{i}^{(\gamma)}\right) \\
& \left.\quad \times\left(\frac{1}{2}-\tau_{j}^{(\gamma)}\right)\right\} .
\end{aligned}
$$

In order to derive magnetic excitations for the systems with orbital degeneracy one usually derives magnetic exchange constants for a bond $\langle i j\rangle$ by averaging over the orbital operators in Eq. (10):

$$
J_{i j}=\left\langle\hat{\mathcal{J}}_{i j}^{(\gamma)}\right\rangle \text {. }
$$

This procedure assumes implicitly that spin and orbital operators can be decoupled from each other and ignores the possibility of entanglement and composite spinorbital excitations introduced in Ref. [5]. It turns out that such excitations play a prominent role in destabilizing the classical AF long-range order in the $d^{9}$ spinorbital model [22].

Qualitatively, the classical phase diagram of model (11) including the crystal-field term (7) consists in the $\left\{\varepsilon_{z}, \eta\right\}$ plane of four AF phases shown schematically in Fig. 1. First, if $\eta$ is small, then: (i) if $\varepsilon_{z}>0$, the order is AF and the holes occupy $|x\rangle$ orbitals (2D AF $x$ phase), while (ii) for $\varepsilon_{z}<0-|z\rangle$ orbitals are occupied in the anisotropic $3 \mathrm{D} \mathrm{AF} z$ phase. Second, if $J_{\mathrm{H}} / U$ is large, the high-spin excitations become important for the bonds with different orbitals on both sites, and weak FM interactions $(\propto \eta J)$ occur within the $a b$ planes, accompanied by strong $(\propto J)$ AF interactions along the $c$-axis, leading to $A$-AF phase. Depending on the actual value of $\varepsilon_{z}$, either (iii) $A$-AF1 phase, or (iv) $A$-AF2 phase (with AF $b c$ planes and FM bonds along the $a$ axis) is stable on the classical level. The energies of all the classical phases are degenerate at the $\left(\varepsilon_{z}, \eta\right)=(0,0)$ point. The competition between the above types of spin and orbital order leads here to the quantum critical point at $\left(\varepsilon_{z}, \eta\right)=(0,0)$.
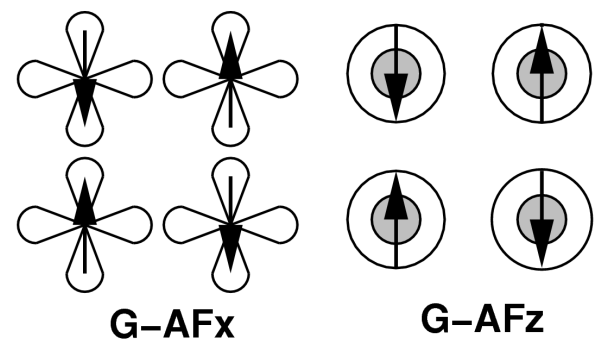

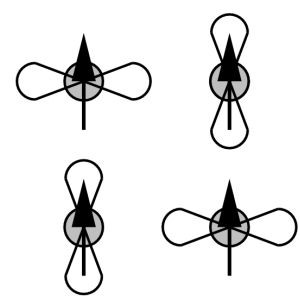

A-AF1

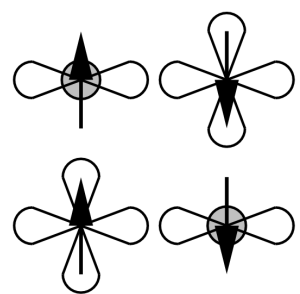

A-AF2
Fig. 1. Schematic representation of orbital and magnetic long-range order in the $a b$ plane of the $d^{9}$ model (11) in: $G$-AF $x, G-\mathrm{AF} z, A-\mathrm{AF} 1$, and $A$-AF2 phase. The shaded parts of different orbitals are oriented along the $c$-axis. The spins (arrows) in the next $a b$ plane along the $c$ axis are AF to those below them in both $G-\mathrm{AF} z$ and $A$-AF 1 phase, and FM in the $A$-AF 2 phase. In the $G$-AF $x$ phase there is no superexchange coupling along the $c$ axis to the next plane, but this degeneracy is easily removed when $|x\rangle$ orbitals mix weakly with $|z\rangle$ orbitals.

It has been argued $[5,22]$ that, similar to the situation in frustrated $J_{1}-J_{2}-J_{3}$ spin models in quantum magnetism where the quantum corrections obtained from the spin-wave theory diverge and suppress the long-range order near quantum critical points [23], the spin order in all the $G$-AF and $A$-AF phases is suppressed near the $\left(\varepsilon_{z} / J, \eta\right)=(0,0)$ point. Although counterarguments were also given that the $\mathrm{AF} z$ (but not $\mathrm{AF} x$ !) phase could be stabilized by order-out-of-disorder mechanism [24] and this controversial issue awaits still an answer with a more sophisticated treatment, it is believed that only short-range order with correlations of valence-bond (VB) type may survive near the transitions between different phases with classical order (Fig. 1). In Ref. [5] a few representative variational VB wave functions (see Fig. 2) were constructed, with spin singlets on individual bonds $\{\langle i j\rangle\}$ accompanied by the variationally optimized orbitals pointing predominantly along the bond. Each VB phase is characterized by a set $K$ which defines 
the bonds $\{\langle i j\rangle\}$ occupied by spin singlets, covering the $3 \mathrm{D}$ lattice. The two simplest (dimer) VB states are (see Fig. 2): (i) singlets along the $a$ axis with orbitals close to $3 x^{2}-r^{2}$ (VBa phase, degenerate with the analogous $\mathrm{VB} b$ phase), (ii) singlets along the $c$-axis with $|z\rangle$ orbitals $(\mathrm{VB} c)$, are indeed more stable than the above classical AF phases, and we emphasize that this happens in three dimensions.
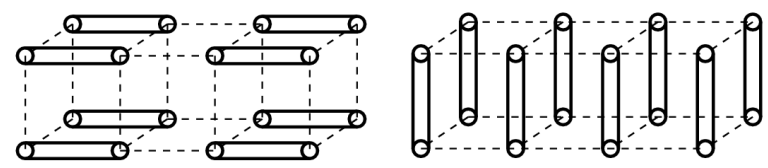

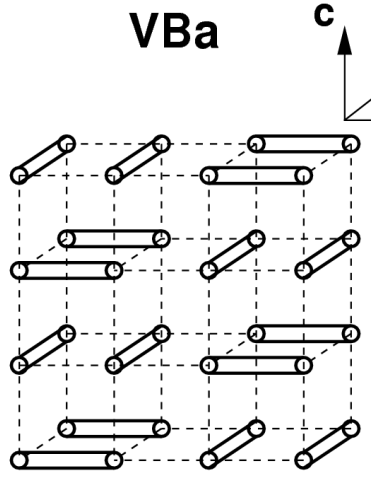

PVBA
VBC

\section{a}

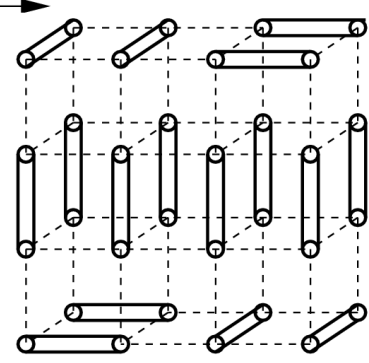

PVBIC
Fig. 2. Spin singlets (double lines) in VB states. The singlets along $a$ axis $(\mathrm{VB} a)$ or along $c$ axis $(\mathrm{VB} c)$ and ordered in either PVBA or PVBIc phase. The axes $\{a, b, c\}$ are shown by arrows.

Further improvement of the energy might be expected by including leading quantum fluctuations in the VB $(\mathrm{VB} a$ and $\mathrm{VB} c$ ) states. In case of the $\mathrm{VB} a$ phase this would lead to resonance on each plaquette between the two components composed of spin singlets along the $a$ and $b$ axis, respectively, but this is hindered by the optimized $z$-like orbitals, oriented in these states either along the $a$, or along the $b$ axis (therefore the overlap between these two configurations is severely reduced). Unlike in the Heisenberg antiferromagnet, the bonds not occupied by singlets contribute finite energy due to the orbital terms similar to those in Sect. 1, and it turns out to be better to optimize the orbital energies due to those bonds depending on whether the bond connects two singlets: either (i) along a single $(a, b$, or $c)$ axis, or (ii) oriented along two different axes with an angle of $\pi / 2$, or (iii) parallel to each other, with the bond making itself an angle of $\pi / 2$ to both of them. As the second type of non-singlet bond is energetically the most favorable one, more energy is gained if plaquettes occupied by singlets along $a$ and $b$ axis alternate and form a 3D superlattice, a plaquette VB alternating (PVBA) state (Fig. 2), giving a lower energy than the quantumcorrected $A$-AF phase. In contrast, the energy of the $\mathrm{VB} c$ phase is decreased more by the resonance between the vertical singlets $(\langle i j\rangle \| c)$. The energy of the result- ing resonating $\mathrm{VB} c(\mathrm{RVB} c)$ state could be obtained using the Bethe ansatz result for the 1D Heisenberg antiferromagnet, and adding the orbital energies due to the bonds $\langle i j\rangle \| a b$.

Furthermore, a PVB interlayered phase along the $c$ axis $(\mathrm{PVBI} c)$, composed of single planes of the PVBA phase interlayered with two planes of $\mathrm{VB} c$ vertical singlets (Fig. 2), is more stable in the crossover regime between the RVBc and PVBA phases for $\varepsilon_{z}<0$. The orbital energies $\propto J$ in the bonds which connect the singlets on the bonds along the $c$ axis with those lying in the $a b$ planes are then optimized, while the energies $\propto \varepsilon_{z}$ are gained in the double layers of $\mathrm{VB} c$ phase. For $\varepsilon_{z}>0$, a rotated interlayered phase (PVBI $a$ ) is more stable before the PVBA takes over. Altogether, one finds [5] that an exotic spin-orbital liquid state, represented here by $\mathrm{RVB} c, \mathrm{PVBI} c, \mathrm{PVBI} a$ and PVBA phases, is favored in an extended regime of parameters, in analogy with a $2 \mathrm{D}$ $1 / 5$-depleted lattice [25], but the present instability is stronger and happens in a $3 \mathrm{D}$ model.

Finally, we remark that the present $d^{9}$ model (11) applies also to $d^{7}$ low-spin $\mathrm{Ni}^{3+}$ ions in $\mathrm{LiNiO}_{2}$ via a particle-hole transformation. However, the situation is rather complex there and also other models were proposed. First, it was argued that a model based on symmetry arguments [26], characterized by a large number of low-lying singlets associated to dimer coverings of the triangular lattice, could explain the properties of $\mathrm{LiNiO}_{2}$. Second, it was shown that charge-transfer terms contribute to the superexchange and they change the balance between different terms in the Hamiltonian, making the orbital interactions stronger than the spin ones [27]. In any case, interplane JT interplane coupling seems to be too weak in $\mathrm{LiNiO}_{2}$ to stabilize the orbital long-range order. The observed difference in the physical properties between $\mathrm{NaNiO}_{2}$ and $\mathrm{LiNiO}_{2}$ remains one of the puzzling phenomena in the field [27] and awaits a future study which has to include all the above aspects.

\section{Entanglement in spin-orbital models}

Future developments in the theory to understand better the nature of ordered and disordered states in spinorbital systems have to take into account possible entanglement between both spin and orbital degrees of freedom on the bonds, as for instance in the states of Fig. 2. It is a common wisdom that the magnetism of correlated Mott insulators can be understood by means of the Goodenough-Kanamori rules. They originate from the mean-field (MF) picture and predict that the superexchange interaction between two magnetic ions with degenerate orbitals is strongly AF because of the Pauli principle if the overlap between the occupied orbitals is large, whereas it is weakly FM when the overlap is weak or virtually disappears. This means that spin order and orbital order are complementary - ferro orbital (FO) order supports AF order, while AO order supports FM spin order. Indeed, these celebrated rules are well followed in 
the manganites $[28,29]$, where the $\mathrm{AO}$ is robust in the FM $a b$ planes, while the orbitals obey the FO order along the $c$ axis, supporting the AF coupling and leading to the $A-\mathrm{AF}$ phase. In fact, one may use here the MF decoupling of the spin and orbital operators, which is sufficient to explain both the magnetic and optical properties of $\mathrm{LaMnO}_{3}$ [8]. The orbital order (or liquid) state determines as well whether the intersite spin correlations are AF or FM in monolayer [30] and in bilayer [31] manganites, while in the perovskite $\mathrm{La}_{1-x} \mathrm{Sr}_{x} \mathrm{MnO}_{3}$ systems the magnons in the FM metallic phase are well explained by the orbital liquid state [32].

As a prominent example of the Goodenough-Kanamori complementarity we would like to mention also the AF phases realized in $\mathrm{YVO}_{3}[33]$, which are the subject of intense research in recent years. While $x y$ orbitals of $\mathrm{V}^{3+}$ ions are occupied due to $\mathrm{GdFeO}_{3}$ distortions, the second electron of the $d^{2}$ ionic configuration may occupy either $y z$ or $z x$ orbital. Depending on whether these orbitals follow FO or AO order along the $c$ axis, the magnetic correlations are there either AF or FM, explaining the origin of the two observed types of AF order shown in Fig. 3: (i) the $C$-AF phase, and (ii) the $G$-AF phase (with AF spin order along all three cubic directions).

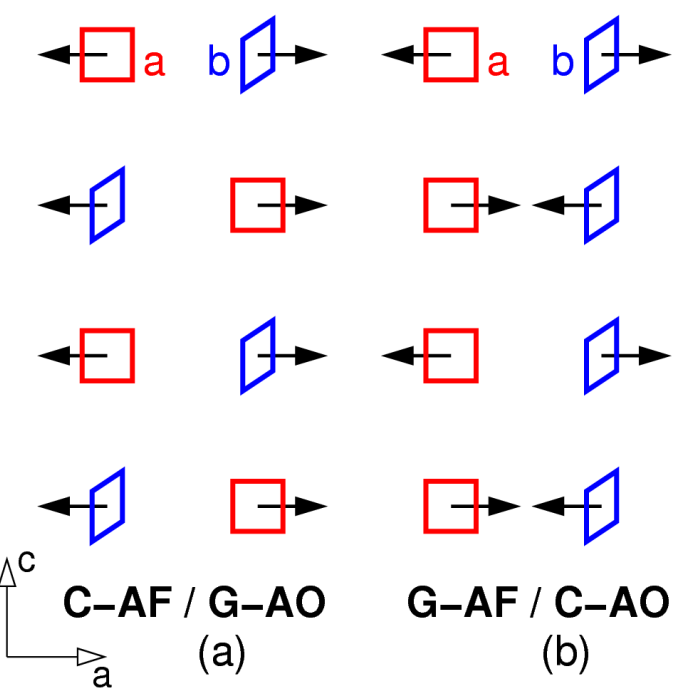

Fig. 3. Goodenough-Kanamori rules on the example of two AF phases observed in the $\mathrm{RVO}_{3}$ perovskites, with complementary magnetic and orbital order in the $a c$ plane: (a) $C$-AF spin order accompanied by $G-\mathrm{AO}$ order; and (b) $G$-AF spin order accompanied by $C$-AO order. Arrows indicate spin order, while squares stand for two active $t_{2 \mathrm{~g}}$ orbitals, $a$ and $b$. Both spins and orbitals alternate along the $b$ axis (not shown).

One may verify the Goodenough-Kanamori rules by evaluating intersite spin and orbital correlations

$$
S_{i j}=\left\langle\boldsymbol{S}_{i} \cdot \boldsymbol{S}_{j}\right\rangle /(2 S)^{2}, \quad T_{i j}=\left\langle\boldsymbol{T}_{i} \cdot \boldsymbol{T}_{j}\right\rangle
$$

and comparing them with each other. In addition, spinorbital entanglement can be measured by the composite correlation function defined for a bond $\langle i j\rangle[6]$ :

$$
C_{i j}=\frac{\left\langle\left(\boldsymbol{S}_{i} \cdot \boldsymbol{S}_{j}\right)\left(\boldsymbol{T}_{i} \cdot \boldsymbol{T}_{j}\right)\right\rangle-\left\langle\boldsymbol{S}_{i} \cdot \boldsymbol{S}_{j}\right\rangle\left\langle\boldsymbol{T}_{i} \cdot \boldsymbol{T}_{j}\right\rangle}{(2 S)^{2}} .
$$

When $C_{i j}=0$, the spin and orbital operators are disentangled and their MF decoupling can be applied, while if $C_{i j}<0-$ spin and orbital operators are entangled. Let us consider again the structure of spin-orbital superexchange models (10) derived for $t_{2 \mathrm{~g}}$ systems from charge excitations between transition metal ions in configurations: $d^{1}$ ( $\mathrm{Ti}^{3+}$ ions in cubic titanates) with $S=1 / 2$ [34], and $d^{2}$ ( $\mathrm{V}^{3+}$ ions in cubic vanadates) with $S=1$ [35] (for more details about the structure of $\mathcal{H}_{J}$ in both $d^{1}$ and $d^{2}$ model see for instance Ref. [8]).

To capture the essence of spin-orbital entanglement it is sufficient to solve $t_{2 \mathrm{~g}}$ models for $d^{1}$ and $d^{2}$ configurations on four-site chains oriented along the $c$ axis using periodic boundary conditions. One finds entangled spin-orbital states in both models, and nontrivial spin-orbital dynamics strongly influences both intersite spin and orbital intersite correlations (13). In the $d^{1}$ (titanate) case one recovers the $\mathrm{SU}(4)$ model with $S_{i j}=T_{i j}=C_{i j}=-0.25$ at $\eta=0$ (Fig. 4a). At finite $\eta$ the $\mathrm{SU}(4)$ degeneracy of all intersite correlations is removed and $T_{i j}<C_{i j}<S_{i j}<0$, as long as the spin singlet $(S=0)$ ground state persists, i.e., for $\eta<0.21$. In this regime the Goodenough-Kanamori rule with complementary correlations (implying different signs of $S_{i j}$ and $T_{i j}$ ), is violated. Instead, by analyzing the values of the intersite correlations one finds that the ground state wave function for each bond $\langle i j\rangle$ is close to a total spinorbital singlet, involving a linear combination of (spin singlet/orbital triplet) and (spin triplet/orbital singlet) states. The vanadate $d^{2}$ model behaves in a similar way, with all three $S_{i j}, T_{i j}$ and $C_{i j}$ correlations being negative in the spin-singlet $(S=0)$ regime of fluctuating $y z$ and $z x$ orbitals obtained for $\eta<0.07$ (Fig. 4b). Therefore, the composite spin-orbital correlations are here finite $\left(C_{i j}<0\right)$, spin and orbital variables are entangled, and the MF factorization of the ground state fails.

To provide further evidence that the GoodenoughKanamori rules do not apply to $t_{2 \mathrm{~g}}$ systems in the regime of small $\eta$, we compare spin exchange constants $J_{i j}$ (12) with the actual values of intersite spin correlations $S_{i j}$ (13). One finds that exchange interaction is formally FM $\left(J_{i j}<0\right)$ in the orbital-disordered phase at low values of $\eta$ (Figs. $4 \mathrm{c}$ and d) is in fact accompanied by AF spin correlations $\left(S_{i j}<0\right)$, so $J_{i j} S_{i j}>0$ and the ground state energy calculated in the MF theory is enhanced [6]. In contrast, similar analysis (not shown) performed for spin-orbital model (11), derived for $d^{9}$ ions with $e_{\mathrm{g}}$ orbital degrees of freedom, gave $J_{i j} S_{i j}<0$, so here spin correlations follow the sign of the exchange constant [6]. This remarkable difference between $t_{2 \mathrm{~g}}$ and $e_{\mathrm{g}}$ systems originates from composite spin-orbital fluctuations, which are responsible for the dynamical nature of exchange constants in the former case. They exhibit large fluctuations around the average value, measured by $\delta J=\left\{\left\langle\left(\hat{\mathcal{J}}_{i j}^{(\gamma)}\right)^{2}\right\rangle-J_{i j}^{2}\right\}^{1 / 2}$. As an illustrative example, we give here the values found in the $d^{1}$ and $d^{2}$ model at 

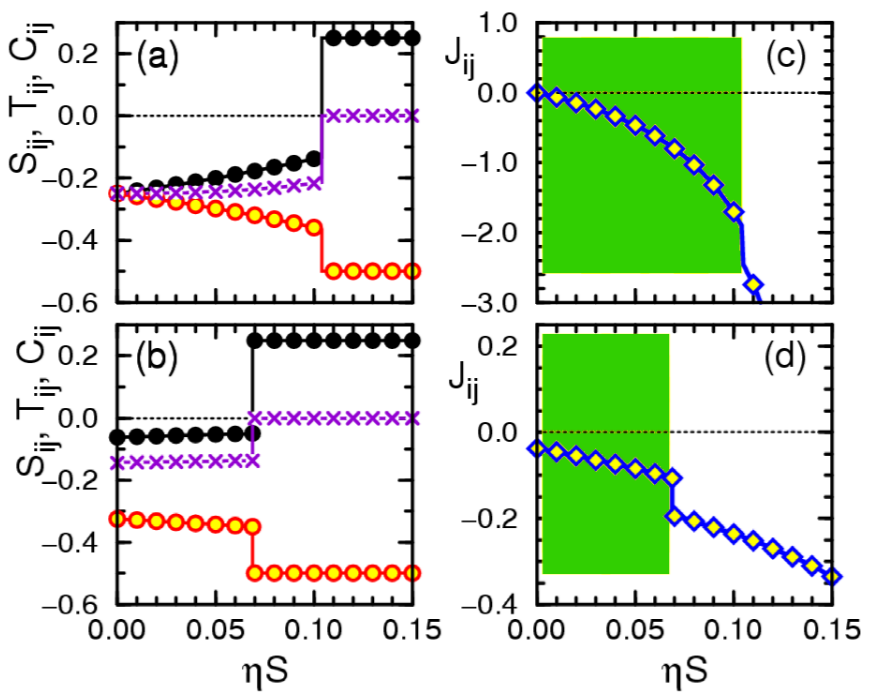

Fig. 4. Left - intersite spin $S_{i j}=\left\langle\boldsymbol{S}_{i} \cdot \boldsymbol{S}_{j}\right\rangle$ (filled circles), orbital $T_{i j}=\left\langle\boldsymbol{T}_{i} \cdot \boldsymbol{T}_{j}\right\rangle$ (empty circles), and composite spin-orbital $C_{i j}$ (14) (crosses) correlations for increasing Hund's exchange $\eta S$ along $c$ axis; right the corresponding spin exchange constants $J_{i j}(14)$, as obtained for $N=4$ site spin-orbital chain (10) with PBC for: (a),(c) $d^{1}$ (titanate, $S=1 / 2$ ) model, and (b),(d) $d^{2}$ (vanadate, $S=1$ ) model. In the shaded areas of (c) and (d) the spin correlations $S_{i j}<0$ do not follow the sign of the exchange constant $J_{i j}<0$, and the classical Goodenough-Kanamori rule is violated.

$\eta=0$. While the average spin exchange constant is small in both cases $\left(J_{i j} \approx 0\right.$ for $d^{1}, J_{i j} \approx-0.04$ for $\left.d^{2}\right), \hat{\mathcal{J}}_{i j}^{(\gamma)}$ fluctuates widely over both positive and negative values. In the $d^{1}$ case the fluctuations between $(S=0 / T=1)$ and $(S=1 / T=0)$ bond wave functions are so large that $\delta J=1$ ! They survive even quite far from the high-symmetry $\mathrm{SU}(4)$ point (at $\eta>0.1$ ), and stabilize spin-orbital singlet phase in a broad regime of $\eta$. Also in the $d^{2}$ model the orbital bond correlations change dynamically from singlet to triplet, resulting in $\delta J>\left|J_{i j}\right|$, with $\delta J=\frac{1}{4}\left\{1-\left(2 T_{i j}+\frac{1}{2}\right)^{2}\right\}^{1 / 2} \approx 0.25$, while these fluctuations are small for $d^{9}$ model (11) with $e_{\mathrm{g}}$ orbitals.

We emphasize that composite spin-orbital fluctuations which occur in spin-orbital entangled states for realistic parameters determine the magnetic and optical properties of titanates and vanadates. For instance, such composite spin-orbital fluctuations are responsible for the temperature dependence of the optical spectral weights in $\mathrm{LaVO}_{3}$ [21] and trigger spin-orbital dimerization in the $C$-AF phase of $\mathrm{YVO}_{3}$ in the intermediate temperature regime (see below) [36]. Remarkably, the observed dimerization in the magnetic excitations may be seen as a signature of entanglement in excited states which become relevant at finite temperature. It is activated by thermal fluctuations in the spin chain [37], which couple to dimerized correlations in the orbital sector.

\section{Phase transitions in the $\mathrm{RVO}_{3}$ perovskites}

Recent progress in experimental studies of transition metal oxides provided exceptionally detailed information on the phase diagrams of the $\mathrm{RMnO}_{3}$ manganites [38] and the $\mathrm{RVO}_{3}$ vanadates [39] (where $\mathrm{R}=\mathrm{La}, \mathrm{Pr}, \ldots, \mathrm{Lu}$ ). In the manganites the orbital order appears first when the temperature is lowered (at $T_{\mathrm{OO}} \approx 800 \mathrm{~K}$ ), and its onset is accompanied by a lattice distortion. The magnetic order follows at much lower temperature $T_{\mathrm{N}} \approx 140 \mathrm{~K}$. This separation of the spin and orbital energy scales follows also from the JT distortions which contribute to the orbital interactions and enhance the value of $T_{\mathrm{OO}}$ [4]. Recent experiments showed that the orbital transition temperature $T_{\mathrm{OO}}$ is enhanced when the ionic radius $r_{\mathrm{R}}$ of the $\mathrm{R}^{3+}$ ions decreases along the $\mathrm{RMnO}_{3}$ perovskites, while $T_{\mathrm{N}}$ is drastically reduced, resulting in the change of magnetic order from the $A$-AF to the $E$-AF phase [38]. This behavior could not be understood until now and is one of the challenges for the theory.

The experimental situation in the cubic vanadates is different and even more complex. On the one hand, the magnetic order in $\mathrm{YVO}_{3}$ is $G$-AF (Fig. 5b) at low temperature, and changes at the first order magnetic transition at $T_{\mathrm{N} 2}=77 \mathrm{~K}$ to the $C$-AF structure, which remains stable up to $T_{\mathrm{N} 1} \approx 116 \mathrm{~K}$. The magnetic transition at $T_{\mathrm{N} 2}$ is particularly surprising as the staggered moments change their direction from approximately parallel to the $c$ axis in the $G$-AF phase to lying almost within the $a b$ planes in the $C$-AF phase, with some small alternating $G$-AF component [40]. Although it was argued that the entropy due to magnetic and orbital excitations is higher in the $C$-AF phase $[35,41]$, the magnetization reversal at the lower magnetic transition remains mysterious, and this phenomenon is still puzzling and far from being completely understood. On the other hand, only the $C$-AF order develops in $\mathrm{LaVO}_{3}$ below a somewhat higher $T_{\mathrm{N} 1} \approx 143 \mathrm{~K}$, and is almost immediately followed by a weak structural transition stabilizing the weak $G$-AO order at $T_{\mathrm{OO}} \approx 141 \mathrm{~K}[39,42]$ (Fig. 5a). Remarkably, the magnetic order parameter in the $C$-AF phase of $\mathrm{LaVO}_{3}$ is strongly reduced to $\approx 1.3 \mu_{\mathrm{B}}$, much below the reduction expected from quantum fluctuations in the $C$-AF phase (being only $6 \%$ for $S=1$ spins) [43] - also this reduction of the measured magnetization could not be explained so far.

Experimental studies have shown that the $C$-AF order is common to the entire family of the $\mathrm{RVO}_{3}$ vanadates, and in general $T_{\mathrm{N} 1}<T_{\mathrm{OO}}$, except for $\mathrm{LaVO}_{3}$ with $T_{\mathrm{N} 1} \approx T_{\mathrm{OO}}[39,42]$. When the ionic radius $r_{\mathrm{R}}$ decreases, the Néel temperature $T_{\mathrm{N} 1}$ also decreases, while orbital transition temperature $T_{\mathrm{OO}}$ increases, passes through a maximum close to $\mathrm{YVO}_{3}$, and decreases again towards $\mathrm{LuVO}_{3}$. Knowing that orbital quantum fluctuations and spin-orbital entanglement play so important role in the perovskite vanadates, it is of interest to ask whether the spin-orbital model of the form (10) introduced for the perovskite vanadates $[35,36]$ is able to describe this variation of $T_{\mathrm{OO}}$ and $T_{\mathrm{N} 1}$ with decreasing radius $r_{\mathrm{R}}$ of $\mathrm{R}$ 
ions in $\mathrm{RVO}_{3}$ [39]. It is clear that the nonmonotonic dependence of $T_{\mathrm{OO}}$ on $r_{\mathrm{R}}$ cannot be reproduced just by the superexchange, as it requires two mechanisms which oppose each other. In fact, there is even no reason to assume that the superexchange constant $J$ should depend on $r_{\mathrm{R}}$, as the distances between $V^{3+}$ ions are quite close to each other in different compounds [44-46], so neither $t$ nor $U$ is expected to change significantly.

As in the $\mathrm{RMnO}_{3}$ manganites [38], one expects that the JT distortions should increase when the ionic radius $r_{R}$ decreases, and one could argue that this would induce the increase in $T_{\mathrm{OO}}$. In order to unravel the physical mechanism responsible for the decrease in $T_{\mathrm{OO}}$ from $\mathrm{YVO}_{3}$ to $\mathrm{LuVO}_{3}$ one has to analyze in more detail the evolution of $\mathrm{GdFeO}_{3}$ distortions with decreasing $r_{\mathrm{R}}$ [47]. Such distortions are common for the perovskites [48], and may be described by two subsequent rotations of $\mathrm{VO}_{6}$ octahedra: (i) by an angle $\vartheta$ around the $b$ axis, and (ii) by an angle $\varphi$ around the $c$ axis. Increasing angle $\vartheta$ causes a decrease in $\mathrm{V}-\mathrm{O}-\mathrm{V}$ bond angles along the $c$ direction, being $\pi-2 \vartheta$, and leads to an orthorhombic lattice distortion $u=(b-a) / a$, where $a$ and $b$ are the lattice parameters of the Pbnm structure of $\mathrm{RVO}_{3}$. The structural data for the perovskite $\mathrm{RVO}_{3}$ vanadates [44-46] give the following empirical relation between the ionic radius $r_{\mathrm{R}}$ and the angle $\vartheta$ :

$$
r_{\mathrm{R}}=r_{0}-\alpha \sin ^{2} \vartheta,
$$

where $r_{0}=1.5 \AA$ and $\alpha=0.95 \AA$. This allows one to use the angle $\vartheta$ to parameterize the dependence of the microscopic parameters and the transition temperatures $T_{\mathrm{OO}}$ and $T_{\mathrm{N} 1}$ on $r_{\mathrm{R}}$.

The spin-orbital model introduced in Ref. [47] to describe the phase diagram of $\mathrm{RVO}_{3}$ includes: (i) the superexchange $\propto J$ between $\mathrm{V}^{3+}$ ions in the $d^{2}$ configuration with $S=1$ spins [35], (ii) the crystal-field splitting $\propto E_{z}$ between $y z$ and $z x$ orbitals, (iii) intersite orbital interactions $\propto V_{a b}, V_{c}$ (which originate from the coupling to the lattice), and (iv) orbital-lattice term which induces orbital polarization when the distortion $u$ increases. The Hamiltonian consists thus of several terms [47]:

$$
\begin{aligned}
\mathcal{H} & =J \sum_{\langle i j\rangle \| \gamma}\left[\left(\boldsymbol{S}_{i} \cdot \boldsymbol{S}_{j}+S^{2}\right) \mathcal{J}_{i j}^{(\gamma)}+\mathcal{K}_{i j}^{(\gamma)}\right] \\
& +E_{z}(\vartheta) \sum_{i} \mathrm{e}^{\mathrm{i} \boldsymbol{R}_{i} \boldsymbol{Q}} \tau_{i}^{z}-V_{c}(\vartheta) \sum_{\langle i j\rangle \| c} \tau_{i}^{z} \tau_{j}^{z} \\
& +V_{a b}(\vartheta) \sum_{\langle i j\rangle \| a b} \tau_{i}^{z} \tau_{j}^{z} \\
& -g u \sum_{i} \tau_{i}^{x}+\frac{1}{2} N K\left[u-u_{0}(\vartheta)\right]^{2},
\end{aligned}
$$

with $\gamma=a, b, c$ labeling the cubic axes. The orbital operators take the form

$$
\begin{aligned}
& \hat{J}_{i j}^{(\gamma)}=\frac{1}{2}\left[\left(1+2 \eta r_{1}\right)\left(\boldsymbol{\tau}_{i} \cdot \boldsymbol{\tau}_{j}+\frac{1}{4} n_{i} n_{j}\right)\right. \\
& \left.\quad-\eta r_{3}\left(\boldsymbol{\tau}_{i} \times \boldsymbol{\tau}_{j}+\frac{1}{4} n_{i} n_{j}\right)-\frac{1}{2} \eta r_{1}\left(n_{i}+n_{j}\right)\right]^{(\gamma)}, \quad(17) \\
& \hat{K}_{i j}^{(\gamma)}=\left[\eta r_{1}\left(\boldsymbol{\tau}_{i} \cdot \boldsymbol{\tau}_{j}+\frac{1}{4} n_{i} n_{j}\right)+\eta r_{3}\left(\boldsymbol{\tau}_{i} \times \boldsymbol{\tau}_{j}+\frac{1}{4} n_{i} n_{j}\right)\right.
\end{aligned}
$$

$$
\left.-\frac{1}{4}\left(1+\eta r_{1}\right)\left(n_{i}+n_{j}\right)\right]^{(\gamma)}
$$

and have a rich structure which originates from the projections of the $d_{i}^{3}$ excited states on the respective eigenstates of $\mathrm{V}^{2+}$ ion. They arise due to the $d_{i}^{2} d_{j}^{2} \rightleftharpoons d_{i}^{3} d_{j}^{1}$ charge excitations, leading either to high-spin or to low-spin $d_{i}^{3}$ configurations, so Hund's exchange splittings in the multiplet structure of $\mathrm{V}^{2+}$ ions enter via the coefficients $r_{1}=1 /(1-3 \eta)$ and $r_{3}=1 /(1+2 \eta)$ (the lower energy singlet excitations occur at energy $U$, so the corresponding coefficient is $r_{2}=1$ ). Using the atomic value of Hund's exchange, we have estimated that $\eta \approx 0.13$ $[8,41]$. The orbital operators $\boldsymbol{\tau}_{i}=\left\{\tau_{i}^{+}, \tau_{i}^{-}, \tau_{i}^{z}\right\}$ for pseudospin $\tau=1 / 2$ in Eqs. (17) and (18) are defined in the subspace spanned by two orbital flavors which are active along a given cubic direction $\gamma=a, b, c$. Unlike in $d^{9}$ model (11) for $e_{\mathrm{g}}$ orbitals, the leading orbital interactions are here proportional to the scalar products $\left(\boldsymbol{\tau}_{i} \cdot \boldsymbol{\tau}_{j}\right)^{(\gamma)}$ of orbital operators on the bonds, but the structure of local Coulomb interactions is responsible for additional terms

$$
\left(\boldsymbol{\tau}_{i} \times \boldsymbol{\tau}_{j}\right)^{(\gamma)}=\frac{1}{2}\left(\tau_{i}^{+} \tau_{j}^{+}+\tau_{i}^{-} \tau_{j}^{-}\right)+\tau_{i}^{z} \tau_{j}^{z},
$$

so the orbital quantum numbers are not conserved. Finally, the operator $n_{i}^{(\gamma)}$ stands for the number of active electrons at site $i$ along the bond $\langle i j\rangle$, for instance for a bond along the $c$ axis this number is $n_{i}^{(c)}=n_{i, y z}+n_{i, z x}$.

Further insight into the electronic configuration on $\mathrm{V}^{3+}$ ions in $\mathrm{RVO}_{3}$ may be obtained either by investigating the electronic structure [49], or by explicit calculations using the point charge model [47]. Both lead to the electronic configurations $(x y)^{1}(y z / z x)^{1}$, i.e., the $x y$ orbitals are occupied at all sites, while the remaining $\{y z, z x\}$ orbitals represent active orbital degrees of freedom which contribute to the $t_{2 \mathrm{~g}}$ orbital dynamics, expressed by the scalar product $\boldsymbol{\tau}_{i} \cdot \boldsymbol{\tau}_{j}$ (only for $\gamma \equiv c$ ). This demonstrates an important difference between the $e_{\mathrm{g}}$ (with one electron or one hole in $e_{\mathrm{g}}$ orbitals at each site) and a $t_{2 \mathrm{~g}}$ system, such as the $\mathrm{RVO}_{3}$ perovskites. Two active $t_{2 \mathrm{~g}}$ orbitals along this bond open a new possibility — if both orbitals are singly occupied, an orbital singlet gives here FM superexchange, even in the absence of Hund's exchange (at $\eta=0$ ) [35].

Furthermore, the actual electronic configurations realized in the $\mathrm{RVO}_{3}$ systems justify the form of the crystal-field term used in Eq. (16), with $\tau_{i}^{z}=\frac{1}{2}\left(n_{i, y z}-n_{i, z x}\right)$. The crystal field alternates in the $a b$ planes, but is uniform along the $c$ axis. It is thus characterized by the vector $\boldsymbol{Q}=(\pi, \pi, 0)$ in reciprocal space, and competes with the (weak) $G$-AO order supporting the observed $C$-AF phase below $T_{\mathrm{N} 1}$. The orbital interactions induced by the distortions of the $\mathrm{VO}_{6}$ octahedra and by $\mathrm{GdFeO}_{3}$ distortions of the lattice, $V_{a b}>0$ and $V_{c}>0$, also favor the $C$-AO order (like $E_{z}>0$ ). Let us note that $V_{c}>0$ counteracts the orbital interactions included in the superexchange. In case of $V_{c}$ one may assume that its dependence on the angle $\vartheta$ is weak, and a constant $V_{c}(\vartheta)=0.26 J$ was chosen in Ref. [47] in order to satisfy the experimental condition that the $C$-AF and $G$-AO order appears almost simultaneously in $\mathrm{LaVO}_{3}$, see Fig. 5a. 


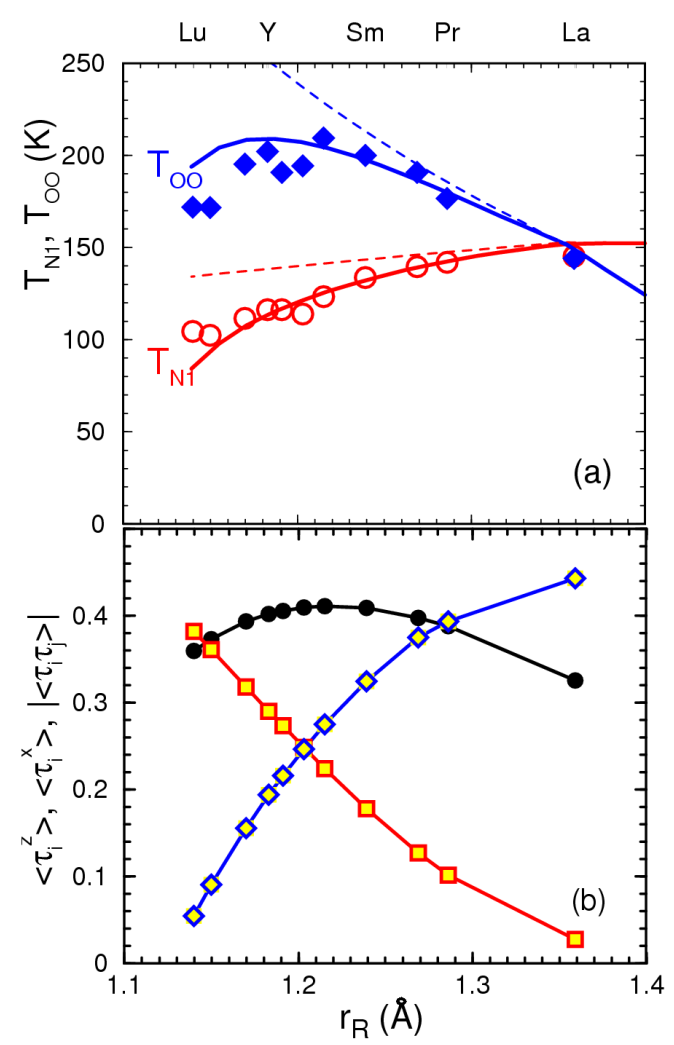

Fig. 5. Theoretical description of orbital and magnetic phase transition in the $\mathrm{RVO}_{3}$ perovskites obtained using model (16), for varying ionic radius $r_{\mathrm{R}}$ of $\mathrm{R}$ ions: (a) transition temperatures $-T_{\mathrm{OO}}$ for the onset of orbital and $T_{\mathrm{N} 1}$ for the onset of the $C$-AF order, as obtained in Ref. [47] (solid lines), and compared with the experimental data of Ref. [39] (filled diamonds and open circles); (b) evolution of orbital order parameter $\left\langle\tau_{i}^{z}\right\rangle_{G}$ (filled circles), transverse orbital polarization $\left\langle\tau_{i}^{x}\right\rangle$ (squares), and orbital intersite correlations $\left|\left\langle\boldsymbol{\tau}_{i} \cdot \boldsymbol{\tau}_{j}\right\rangle\right|$ (diamonds) along $c$ axis at $T=0$. Parameters: $J=200 \mathrm{~K}$, $v_{z}=17, v_{a b}=22, v_{\mathrm{g}}=740$.

The experimental value $T_{\mathrm{N} 1}^{\exp }=143 \mathrm{~K}$ for $\mathrm{LaVO}_{3}$ [39] was fairly well reproduced in the present model taking $J=200 \mathrm{~K}$. The functional dependence of the remaining two parameters $\left\{E_{z}, V_{a b}\right\}$ on the tilting angle $\vartheta$ was derived from the point charge model [47], using the structural data for $\mathrm{RVO}_{3}$ [44-46]. In this way the following two relations were deduced:

$$
\begin{aligned}
& E_{z}(\vartheta)=J v_{z} \sin ^{3} \vartheta \cos \vartheta, \\
& V_{a b}(\vartheta)=J v_{a b} \sin ^{3} \vartheta \cos \vartheta .
\end{aligned}
$$

The last two terms in Eq. (16) describe the linear coupling $\propto g>0$ between active $\{y z, z x\}$ orbitals and the orthorhombic lattice distortion $u$. The elastic energy which counteracts lattice distortion $u$ is given the force constant $K$, and $N$ is the number of $\mathrm{V}^{3+}$ ions. The coupling $\propto g u$ acts as a transverse field in the pseudospin space. While the eigenstates $\frac{1}{\sqrt{2}}(|y z\rangle \pm|z x\rangle)$ favored by $\tau_{i}^{x}$ cannot be realized due to the competition with all the other terms, increasing lattice distortion $u$ (increasing angle $\vartheta$ ) modifies the orbital order and intersite orbital correlations. At the minimum one finds an effective coupling

$$
g_{\text {eff }}(\vartheta ; T) \equiv g u(\vartheta ; T)=g u_{0}(\vartheta)+\frac{g^{2}}{K}\left\langle\tau^{x}\right\rangle_{T},
$$

with the global distortion $u(\vartheta ; T)$ caused by (i) a pure lattice term $u_{0}(\vartheta)$, and (ii) an orbital contribution $\propto\left\langle\tau^{x}\right\rangle$ which can be determined self-consistently within the present approach, see Ref. [47]. Both $u_{0}$ and $\left\langle\tau^{x}\right\rangle$ are expected to increase with increasing tilting $\vartheta$. This dependence has to be faster than the ones of Eqs. (20) and (21), as otherwise no maximum in the dependence of $T_{\mathrm{OO}}$ on $r_{\mathrm{R}}$ would be obtained. Using this argument a semiempirical relation

$$
g_{\text {eff }}(\vartheta)=J v_{\mathrm{g}} \sin ^{5} \vartheta \cos \vartheta
$$

was postulated in Ref. [47]. Therefore, magnetic and orbital correlations described by spin-orbital model (10) depend on three parameters: $\left\{v_{z}, v_{a b}, v_{\mathrm{g}}\right\}$.

While the above fast dependence on the tilting angle $\vartheta$ of $\mathrm{VO}_{6}$ octahedra in the $\mathrm{RVO}_{3}$ family was introduced in order to reproduce the experimentally observed dependence of $T_{\mathrm{OO}}$ on $r_{\mathrm{R}}$, see Fig. $5 \mathrm{a}$, it may be justified a posteriori. It turns out that the dependence of $g_{\text {eff }}$ on the ionic radius $r_{\mathrm{R}}$ follows the actual lattice distortion $u$ in $\mathrm{RVO}_{3}$ measured at $T=0$ (or just above $\left.T_{\mathrm{N} 1}\right)$ [47]. Also the orbital polarization $\left\langle\tau^{x}\right\rangle$ is approximately $\propto \sin ^{5} \vartheta \cos \vartheta$, and follows the same fast dependence of $g_{\text {eff }}(\vartheta)$ for the $\mathrm{RVO}_{3}$ perovskites. These results indicate that the bare coupling parameters $\{g, K\}$ are nearly constant and independent of $r_{\mathrm{R}}$, which may be treated as a prediction of the theory to be verified by future experiments.

Similar to the $\mathrm{RMnO}_{3}$ manganites [4], the correct $\mathrm{MF}$ treatment of the orbital and magnetic phase transitions in the $\mathrm{RVO}_{3}$ vanadates requires the coupling between the on-site orbital, $\left\langle\tau^{z}\right\rangle_{G} \equiv \frac{1}{2}\left|\left\langle\tau_{i}^{z}-\tau_{j}^{z}\right\rangle\right|$, and spin, $\left\langle S_{i}^{z}\right\rangle_{C}$, order parameters in the $C$-AF phase, as well as a composite $\left\langle S_{i}^{z} \tau_{i}^{z}\right\rangle$ order parameter. Unlike in the perovskite manganites [38], however, the on-site MF theory does not suffice for the $\mathrm{RVO}_{3}$ compounds as the orbital singlet correlations $\left\langle\boldsymbol{\tau}_{i} \cdot \boldsymbol{\tau}_{j}\right\rangle$ on the bonds $\langle i j\rangle$ along the $c$ axis play an important role in stabilizing the $C$-AF phase $[35,41]$. Therefore, the minimal physically acceptable approach to the present problem is a self-consistent calculation for a bond $\langle i j\rangle$ along the $c$ axis, embedded in the MF terms due to its neighbors along all the cubic axes [47]. This procedure, with properly selected model parameters, served to reproduce the experimental phase diagram of Fig. 5a. One finds that the orbital transition occurs first in the $\mathrm{RVO}_{3}$ vanadates with $r_{\mathrm{R}}<r_{\mathrm{La}}$, as observed. At the same time, the Néel temperature $T_{\mathrm{N} 1}$ decreases with decreasing $r_{\mathrm{R}}$.

The key feature of the present spin-orbital system which drives the observed dependence of $T_{\mathrm{OO}}$ and $T_{\mathrm{N} 1}$ on the ionic radius $r_{\mathrm{R}}$ is the evolution of intersite orbital correlations $\left\langle\boldsymbol{\tau}_{i} \cdot \boldsymbol{\tau}_{j}\right\rangle$ along the $c$ axis, and the increasing 
orbital polarization $\left\langle\tau^{x}\right\rangle$ with decreasing $r_{\mathrm{R}}$ (Fig. 5b). Indeed, the singlet correlations are drastically suppressed from $\mathrm{LaVO}_{3}$ towards $\mathrm{LuVO}_{3}$. While $\left\langle\tau^{x}\right\rangle \approx 0.03$ is rather weak in $\mathrm{LaVO}_{3}$, it steadily increases along the $\mathrm{RVO}_{3}$ perovskites when $r_{\mathrm{R}}$ decreases, and finally it becomes as important as the orbital order parameter, $\left\langle\tau^{x}\right\rangle \approx\left\langle\tau^{z}\right\rangle_{G}$. Let us note that in all the cases the latter order parameter is substantially reduced from the classical value $\frac{1}{2}$ by singlet orbital fluctuations, being $\left\langle\tau^{z}\right\rangle_{G} \approx 0.32$ and 0.36 for $\mathrm{LaVO}_{3}$ and $\mathrm{LuVO}_{3}$, respectively.

The results presented in Ref. [47] demonstrate the remarkable dependence of both spin and orbital order on the orbital correlations. First, the increase in orbital intersite interactions due to the JT term (21), induces steady increase in the orbital temperature $T_{\mathrm{OO}}$ with decreasing $r_{\mathrm{R}}$. When the orbital polarization $\left\langle\tau^{x}\right\rangle$ becomes large, however, this increase is suppressed and one reproduces the nonmonotonic dependence of $T_{\mathrm{OO}}$ on $r_{\mathrm{R}}$, with the observed drop of $T_{\mathrm{OO}}$ when $r_{\mathrm{R}}$ decreases beyond $r_{\mathrm{R}} \approx 1.18 \AA$ of $\mathrm{YVO}_{3}$. Second, the changes in intersite orbital correlations shown in Fig. 5b modify the magnetic exchange constants $\left\{J_{a b}, J_{c}\right\}$ along the bonds parallel to one of cubic directions in the $a b$ planes or along the $c$ axis (12), and thus the value of $T_{\mathrm{N} 1}$ is reduced with decreasing $r_{\mathrm{R}}$, although the superexchange energy $J$ does not change. This also means that the width of the magnon band given at $T=0$ by $W_{C-\mathrm{AF}}=4\left(J_{a b}+\left|J_{c}\right|\right)[41]$ is reduced by a factor close to 1.8 from $\mathrm{LaVO}_{3}$ to $\mathrm{YVO}_{3}$, in agreement with surprisingly low magnon energies observed in the $C$-AF phase of $\mathrm{YVO}_{3}$ [33].

Summarizing, spin-orbital model (16) of Ref. [47] provides an almost quantitative understanding of the systematic experimental trends for both orbital and magnetic transitions in the $\mathrm{RVO}_{3}$ perovskites, and is able to reproduce the nonmonotonic variation of the orbital temperature $T_{\mathrm{OO}}$ for decreasing $r_{\mathrm{R}}$. Hovever, the theoretical description of the magnetic transition to the $G$-AF phase at $T_{\mathrm{N} 2}$, which occurs for small $r_{\mathrm{R}}$ [39], remains to be addressed by future theory. More open issues and future directions of reasearch in the field of the orbital physics are shortly indicated in the next section.

\section{Summary and open problems}

A few representative problems discussed above demonstrate that the spin-orbital physics is a very rich field, with intrinsically frustatred interactions and rather exotic ordered or disordered phases, and with their behavior frequently dominated by quantum fluctuations. While valuable information about the electronic structure is obtained from density functional theory [50], the many-body aspects have to be studied using models of correlated electrons. Perhaps the most important feature of orbital or spin-orbital superexchange models is strong coupling between the orbitals and the lattice, which may help to stabilize orbital order, as in model (16) introduced for the $\mathrm{RVO}_{3}$ perovskites [47]. In this way, the lattice distortions may also indirectly influence the onset of magnetic order in systems with active orbital degrees of freedom. If they are absent and the lattice is frustrated in addition, a very interesting situation arises, with strong tendency towards truly exotic quantum states [51]. Examples of this behavior were considered recently for the triangular lattice, both for $e_{\mathrm{g}}$ orbitals in $\mathrm{LiNiO}_{2}$ [27] and $t_{2 \mathrm{~g}}$ orbitals in $\mathrm{NaTiO}_{2}$ [52]. None of these models could really be solved, but generic tendency towards dimer correlations with spin singlets on the bonds for particular orbital states could be shown.

Rapid progress in the field of orbital physics results mainly from developing new experimental techniques and synthesizing novel materials. While the experiment is ahead of the theory in most cases, there are some exceptions. One of them was a theoretical prediction of the energy and dispersion of orbital excitations $[10,53]$. Only recently orbital excitations (orbitons) could be observed in the Raman scattering in the Mott insulators $\mathrm{LaTiO}_{3}$ and $\mathrm{YTiO}_{3}$ [54]. An interesting question which arises in this context is carrier propagation in a Mott insulator with AO order. This problem is very complex and has been addressed also in the last decade of the previous century [55]. Not only spin excitations may provide more final states, as for triplet excitations in the $t-J$ model [56], but a carrier may dress by orbital excitations [57], and scatter on magnons [58]. Indeed, the coupling to orbitons increases the effective mass of a moving hole in $e_{\mathrm{g}}$ systems [57]. The orbital part of the superexchange is classical $t_{2 \mathrm{~g}}$ systems, but also there weak quasiparticle dispersion arises from three-site processes [59] (for more details see also recent Ref. [60]).

Similar to doped manganites [61], also in doped $\mathrm{R}_{1-x}(\mathrm{Sr}, \mathrm{Ca})_{x} \mathrm{VO}_{3}$ systems orbital order gradually disappears [62]. The composite $C$-AF/G-AO order survives, however, is a broad range of doping, in contrast to $\mathrm{La}_{1-x} \mathrm{Sr}_{x} \mathrm{MnO}_{3}$, where $\mathrm{FM}$ order sets in already at $x \approx 0.10$. It is quite remarkable that the complementary $G$-AF $/ C$-AO type of order is fragile and disappears in $\mathrm{Y}_{1-x} \mathrm{Ca}_{x} \mathrm{VO}_{3}$ already at $x=0.02$ [62]. We remark that the holes doped in $C-\mathrm{AF} / G$-AO phase are localized in polaron-like states [63], so the purely electronic model such as of Ref. [59] is too crude to capture both the evolution of the spin-orbital order in doped vanadates and the gradual decrease in the energy scale for spin-orbital fluctuations.

As a final remark, we would like to mention very promising recent experimental studies of Ni-based superlattices [64]. Recent theory for $\mathrm{LaNiO}_{3} / \mathrm{LaMO}_{3}$ superlattices (with $\mathrm{M}=\mathrm{Al}, \mathrm{Gd}, \mathrm{Ti}$ ) [65] predicts that the correlated $e_{\mathrm{g}}$ electrons in the $\mathrm{NiO}_{2}$ planes develop a planar $x^{2}-y^{2}$ orbital order driven by the reduced dimensionality and further supported by epitaxial strain from the substrate. This resembles the $x^{2}-y^{2}$ orbital polarization in doped layered manganites [66]. As in all other cases discussed above, the superexchange interactions which involve the orbital degrees of freedom play here a crucial role to understand the observed magnetic order and low-lying excited states. 


\section{Acknowledgments}

It is a great pleasure to thank all my collaborators, in particular P. Horsch, L.F. Feiner, G. Khaliullin and J. Zaanen, for numerous insightful discussions which contributed to my present understanding of the subject. We acknowledge support by the Foundation for Polish Science (FNP), and by the Ministry of Science and Higher Education under project No. N202 068 32/1481.

\section{References}

[1] M. Imada, A. Fujimori, Y. Tokura, Rev. Mod. Phys. 70, 1039 (1998).

[2] K.I. Kugel, D.I. Khomskii, Sov. Phys. Usp. 25, 231 (1982).

[3] C. Castellani, C.R. Natoli, J. Ranninger, Phys. Rev. B 18, 4945 (1978); 18, 4967 (1978); 18, 5001 (1978).

[4] L.F. Feiner, A.M. Oleś, Phys. Rev. B 59, 3295 (1999).

[5] L.F. Feiner, A.M. Oleś, J. Zaanen, Phys. Rev. Lett. 78, 2799 (1997).

[6] A.M. Oleś, P. Horsch, L.F. Feiner, G. Khaliullin, Phys. Rev. Lett. 96, 147205 (2006).

[7] B. Keimer, A.M. Oleś, Focus on Orbital Physics, New J. Phys. 6, E05 (2004).

[8] A.M. Oleś, P. Horsch, G. Khaliullin, L.F. Feiner, Phys. Rev. B 72, 214431 (2005).

[9] K.A. Chao, J. Spałek, A.M. Oleś, J. Phys. C 10, L271 (1977).

[10] J. van den Brink, F. Mack, P. Horsch, A.M. Oleś, Phys. Rev. B 59, 6795 (1999).

[11] A.M. Oleś, L.F. Feiner, J. Zaanen, Phys. Rev. B 61, $6257(2000)$

[12] J. van den Brink, New J. Phys. 6, 201 (2004).

[13] L.F. Feiner, A.M. Oleś, Phys. Rev. B 71, 144422 (2005).

[14] D.I. Khomskii, M.V. Mostovoy, J. Phys. A 36, 9197 (2003).

[15] J. Dorier, F. Becca, F. Mila, Phys. Rev. B 72, 024448 (2005).

[16] S. Wenzel, W. Janke, Phys. Rev. B 78, 064402 (2008).

[17] L. Longa, A.M. Oleś, J. Phys. A 13, 1031 (1980).

[18] W. Brzezicki, J. Dziarmaga, A.M. Oleś, Phys. Rev. $B$ 75, 134415 (2007).

[19] J.H.H. Perk, H.W. Campel, G.R.W. Quispel, F.W. Nijhoff, Physica A 123, 1 (1984).

[20] W. Brzezicki, A.M. Oleś, Acta Phys. Pol. A 115, 162 (2009).

[21] G. Khaliullin, P. Horsch, A.M. Oleś, Phys. Rev. B 70, 195103 (2004)

[22] L.F. Feiner, A.M. Oleś, J. Zaanen, J. Phys., Condens. Matter 10, L555 (1997).

[23] P. Chandra, B. Douçot, Phys. Rev. B 38, 9335 (1988).

[24] G. Khaliullin, V. Oudovenko, Phys. Rev. B 56 14243(R) (1997).
[25] K. Ueda, H. Kontani, M. Sigrist, P.A. Lee, Phys. Rev. Lett. 76, 1932 (1996).

[26] F. Vernay, K. Penc, P. Fazekas, F. Mila, Phys. Rev. $B$ 70, 014428 (2004).

[27] A.J.W. Reitsma, L.F. Feiner, A.M. Oleś, New J. Phys. 7, 121 (2005).

[28] A. Weiße, H. Fehske, New J. Phys. 6, 158 (2004).

[29] E. Dagotto, New J. Phys. 7, 67 (2005).

[30] M. Daghofer, W. von der Linden, A.M. Oleś, Phys. Rev. B 70, 184430 (2004).

[31] M. Daghofer, A.M. Oleś, D.M. Neuber, W. von der Linden, Phys. Rev. B 73, 104451 (2006).

[32] A.M. Oleś, L.F. Feiner, Phys. Rev. B 65, 052414 (2002).

[33] C. Ulrich, G. Khaliullin, J. Sirker, M. Reehuis, M. Ohl, S. Miyasaka, Y. Tokura, B. Keimer, Phys. Rev. Lett. 91, 257202 (2003).

[34] G. Khaliullin, S. Maekawa, Phys. Rev. Lett. 85, 3950 (2000).

[35] G. Khaliullin, P. Horsch, A.M. Oleś, Phys. Rev. Lett. 86, 3879 (2001).

[36] P. Horsch, G. Khaliullin, A.M. Oleś, Phys. Rev. Lett. 91, 257203 (2003).

[37] J. Sirker, A. Herzog, A.M. Oleś, P. Horsch, Phys. Rev. Lett. 101, 157204 (2008).

[38] J.-S. Zhou, J.B. Goodenough, Phys. Rev. Lett. 96 247202 (2006).

[39] S. Miyasaka, Y. Okimoto, M. Iwama, Y. Tokura, Phys. Rev. B 68, 100406 (2003).

[40] Y. Ren, T.T.M. Palstra, D.I. Khomskii, A.A. Nugroho, A.A. Menovsky, G.A. Sawatzky, Phys. Rev. $B$ 62, 6577 (2000).

[41] A.M. Oleś, P. Horsch, G. Khaliullin, Phys. Rev. B 75, 184434 (2007).

[42] S. Miyasaka, J. Fujioka, M. Iwama, Y. Okimoto, Y. Tokura, Phys. Rev. B 73, 224436 (2006).

[43] M. Raczkowski, A.M. Oleś, Phys. Rev. B 66, 094431 (2002).

[44] M. Reehuis, C. Ulrich, P. Pattison, B. Ouladdiaf, M.C. Rheinstdter, M. Ohl, L.P. Regnault, M.Miyasaka, Y. Tokura, B. Keimer, Phys. Rev. B 73, 094440 (2006).

[45] M.H. Sage, G.R. Blake, G.J. Nieuwenhuys, T.T.M. Palstra, Phys. Rev. Lett. 96, 036401 (2006).

[46] M.H. Sage, G.R. Blake, C. Marquina, T.T.M. Palstra, Phys. Rev. B 96, 195102 (2007).

[47] P. Horsch, A.M. Oleś, L.F. Feiner, G. Khaliullin, Phys. Rev. Lett. 100, 167205 (2008).

[48] E. Pavarini, Y. Yamasaki, J. Nuss, O.K. Andersen, New J. Phys. 7, 188 (2005).

[49] M. De Raychaudhury, E. Pavarini, O.K. Andersen, Phys. Rev. Lett. 99, 126402 (2007).

[50] I.V. Solovyev, J. Phys., Condens. Matter 20, 293201 (2008).

[51] G. Khaliullin, Prog. Theor. Phys. Suppl. 160, 155 (2005).

[52] B. Normand, A.M. Oleś, Phys. Rev. B 78, 094427 (2008) 
[53] J. van den Brink, Phys. Rev. Lett. 87, 217202 (2001).

[54] C. Ulrich, A. Gössling, M. Grüninger, M. Guennou, H. Roth, M. Cwik, T. Lorenz, G. Khaliullin, B. Keimer, Phys. Rev. Lett. 97, 157401 (2006).

[55] J. Zaanen, A.M. Oleś, Phys. Rev. B 48, 7197 (1993).

[56] J. Zaanen, A.M. Oleś, P. Horsch, Phys. Rev. B 46, 5798 (1992).

[57] J. van den Brink, P. Horsch, A.M. Oleś, Phys. Rev. Lett. 85, 5174 (2000).

[58] J. Bała, G.A. Sawatzky, A.M. Oleś, A. Macridin, Phys. Rev. Lett. 87, 067204 (2001).

[59] M. Daghofer, K. Wohlfeld, A.M. Oleś, E. Arrigoni, P. Horsch, Phys. Rev. Lett. 100, 066403 (2008).

[60] K. Wohlfeld, A.M. Oleś, M. Daghofer, P. Horsch, Acta Phys. Pol. A 115, 110 (2009); K. Wohlfeld, A.M. Oleś, M. Daghofer, P. Horsch, Phys. Rev. B 78, 214423 (2008).
[61] M. Daghofer, A.M. Oleś, Acta Phys. Pol. A 111, 497 (2007).

[62] J. Fujioka, S. Miyasaka, Y. Tokura, Phys. Rev. B 72, 024460 (2005).

[63] J. Fujioka, S. Miyasaka, Y. Tokura, Phys. Rev. B 77, 144402 (2008).

[64] J. Chakhalian, J.W. Freeland, G. Srajer, J. Strempfer, G. Khaliullin, J.C. Cezar, T. Charlton, R. Dalgliesh, C. Bernhard, G. Cristiani, H.U. Habermeier, B. Keimer, Nature Phys. 2, 244 (2006).

[65] J. Chaloupka, G. Khaliullin, Phys. Rev. Lett. 100 , 016404 (2008).

[66] F. Mack, P. Horsch, Phys. Rev. Lett. 82, 3160 (1999). 